\author{
Military Technical College \\ Kobry El-Kobbah, \\ Cairo, Egypt
}

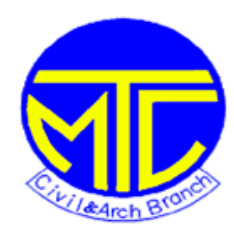

\author{
$11^{\text {th }}$ International Conference \\ on Civil and Architecture \\ Engineering \\ ICCAE-11-2016
}

\title{
A SIMULATION MODEL FOR U-TURN TRAFFIC CAPACITY
}

\author{
HASSAN T. ABDELWAHAB ${ }^{1}$
}

\begin{abstract}
One of the common traffic management techniques for left turns in Cairo urban intersections is replacing direct left-turns with U-turns; basically to eliminate direct left turn movements at the intersection. Currently there is a lack of models that estimate the capacity of U-turns at median openings. Congested U-turns make traffic traveling very slowly and create some opportunities for vendors which further slowing down the already congested vehicular traffic. The objective of this paper is to develop a simulation model to determine capacity of U-turns at uncontrolled mid-block median openings with no police control. The proposed model is based on the gap acceptance process. Results showed when the median width was comfortably sufficient; it leaded to a higher U-turn capacity. The U-turn movement at a wide median can reach a maximum capacity of about $985 \mathrm{veh} / \mathrm{hr}$ when there is no conflicting traffic. The capacity of U-turn decreases as the conflicting volume increases. The capacity is reduced to $540 \mathrm{veh} / \mathrm{hr}$ and $380 \mathrm{veh} / \mathrm{hr}$ when the conflicting volume is 1000 and 1500 veh/hr per lane.
\end{abstract}

\section{Introduction}

U-turns are increasingly being used in Cairo to eliminate the left turns and through movements from the side streets. Motorists desiring to make left turns and through movements from the side-street approaches must make these movements indirectly, via a right turn from the side street, followed by a U-turn at a median break located downstream of the subject intersection. This maneuver often can consume

\footnotetext{
${ }^{1}$ Associate Professor, Public works Department, College of Engineering, Cairo University Mailing Address: Cairo University, College of Engineering, Giza, 12411, Egypt

Email Address: Hassantahsin@yahoo.com; hassan.tahsin@cu.edu.eg

Phone: 01011226110
} 
about a kilometer or sometimes several kilometers before a vehicle is headed in the correct direction. In some instances, drivers try to find shortcuts in an attempt to save their travel time.

A traffic congestion study was concerned with identifying the causes, types and locations of road congestion in Cairo [1]. The study raised operational concerns from the excessive use of U-turn as a dominant access management strategy. U-turn carrying high traffic volumes and lacking deceleration lanes causes traffic turbulences. At some U-turn locations, congested bumper-to-bumper traffic makes travel very slow and it creates some opportunities for vendors. Despite the poor performance, the use of median opening U-turn is increasing in popularity among the city officials in Cairo [2]. The U-turn movement at a median opening is highly complex compared with turning movements at intersections because the turning vehicle has to make a 180-deg movement and join the traffic stream in which it is seeking an acceptable gap. The turning vehicle must wait and then turn under low speed conditions in the face of oncoming traffic and may need to accelerate rapidly to reach the speed of the traffic stream. The average delay for U-turn movements at a median opening is significantly influenced by the conflicting traffic flow [3]. If there are many turning vehicles that have to wait, then a long queue in the stream cannot be avoided and queue spill-back to block traffic is possible. This can lead to traffic problems, mainly reduced capacity [4]. Furthermore, some U-turn vehicles often do not wait for the large enough acceptable gap of the through traffic. They gradually move onto the conflicting lane to show the intention to go and yet create more road hazards and dangerous driving conditions. The through vehicles sometimes do not allow for U-turn, by increasing speed or honking car horn or opening headlight. Eventually, the through traffic stops and allows the U-turn traffic to move [5].

The Egyptian code of practice for urban and rural road provides minimum median width for median openings for different types of U-turn maneuvers. However, traffic capacity of U-turn median openings has not yet been addressed although there are a lot of midblock U-turn facilities on arterials in Cairo. Thus a determination of the capacity of a U-turn median opening is needed. Knowing the capacity of median opening U- 
turn junction leads to the better traffic operation management as well as facilitates the level of service assessment. The primary objective of this paper is the development of a microscopic traffic simulation model to evaluate the capacity and operational performance of median openings serving U-turn traffic. The proposed simulation model enables prediction the operation characteristics of a median opening U-turn facility prior to its construction.

\section{Proposed model logic and experimental design}

\subsection{Model Logic}

When the vehicle arrives at the median opening, its driver faces the gaps of the conflicting through traffic, waiting for an acceptable gap, and then makes U-turn. However, when the conflicting traffic volume is high, it is very difficult to find a large enough gap for the U-turn movement. The proposed model is based on the concept of gap acceptance commonly used in the analysis of un-signalized junctions. After arriving the median opening, the U-turn vehicles wait for a large enough gap to make the U-turn maneuver. A U-turn driver $i$ is willing to accept a gap, $t$, if it is greater than a common critical gap, $T_{c}$, or reject if it is not. The proposed form of the gap acceptance function is a step function $\alpha_{i}(t)$ as follows:

$$
\alpha_{i}(t)= \begin{cases}1 & t \geq T_{c} \\ 0 & t<T_{c}\end{cases}
$$

According to the proposed gap acceptance function, no driver will enter the major traffic stream unless the gap between vehicles in is at least equal to the critical gap, $T_{c}$. The U-turn driver must perceive distances and velocities of the major traffic stream and he/she must have a feeling for his/her own car's performance. For the queued Uturn movement, the followed U-turn vehicles require lesser critical gap, which is called follow-up headway $T_{s}$. The follow-up time is the time between the departure of one vehicle from the minor street and the departure of the next vehicle using the same major-street gap, under a condition of continuous queuing on the minor street. So, if the gap size is between $T_{c}$ and $T_{c}+n T_{f}$, only $n$ vehicles from the queued U-turn 
movement can make the U-turn. For instance, if the gap size is between $T_{c}$ and $T_{c}+2 T_{f}$ then two vehicles can make U-turn. Note the follow-up time is the headway that can be used to define the saturation flow rate of the U-turn if there are no conflicting vehicles. A recent study reported that the follow-up headway is 3.4 seconds [6].

\subsubsection{Headway Distributions}

A large number of headway distributions have been developed to represent the different pattern of vehicle arrivals. The most widely applied assumption is that vehicles arrive randomly and the headways follow exponential distribution [7]. In order to carry out a simulation using random inputs such as headways, probability distributions must be specified. In the proposed simulation model, sequences of random points in time for vehicle arrivals were generated. For instance, the headway times are commonly represented by an exponential random variable with a mean $\bar{h}>0$. The following inverse-transform algorithm can be used to generate the vehicular headway times.

$$
\begin{gathered}
h=-\bar{h} \ln u \\
t_{i}=\sum_{1}^{i} h
\end{gathered}
$$

where $u \sim U(0,1)$ is the distribution function of a uniform random variable having a range $[0,1], h$ is a generated headway instant in seconds, $\bar{h}$ is the average headway in seconds, and $t_{i}$ is the arrival time of a vehicle $i$. The reader is referred to [8] for a detailed discussion about simulation models, generating random variables, variancereduction techniques, and common random numbers. Approaching vehicles are assumed to be uniformly distributed among the basic highway lanes.

\subsubsection{U-turn Maneuver}

One of the key factors in the operation of U-turn is the turning radius which is controlled by the combination of the median nose width and the width of the receiving lanes. The Egyptian code of practice for urban and rural road provides minimum 
median width for median openings for different types of design vehicles as well as minimum turning paths of design vehicles. For a satisfactory design for U-turn maneuvers, the width of the road, including the median, should be sufficient to permit the design vehicle to turn from an auxiliary left-turn lane in the median into the lane next to the outside shoulder.

Figure 1 illustrates the AASHTO minimum turning path for passenger cars. The principal dimensions affecting the turning path are the minimum centerline turning radius, the out-to-out track width, the wheelbase, and the path of the inner rear tire. Effects of driver characteristics (such as the speed at which the driver makes a turn and the slip angles of wheels) are minimized when the speed of the vehicle for the minimum turning radius is less than $15 \mathrm{~km} / \mathrm{h}$. To be able to make a U-turn to the inner lane, the median width should be at least $8.78 \mathrm{~m}$, which is double the radius path of the diameter of the right rear wheel overhang. Trucks and buses generally need more generous geometric designs than do passenger vehicles. This is largely because trucks and buses are wider and have longer wheelbases and greater turning radii.

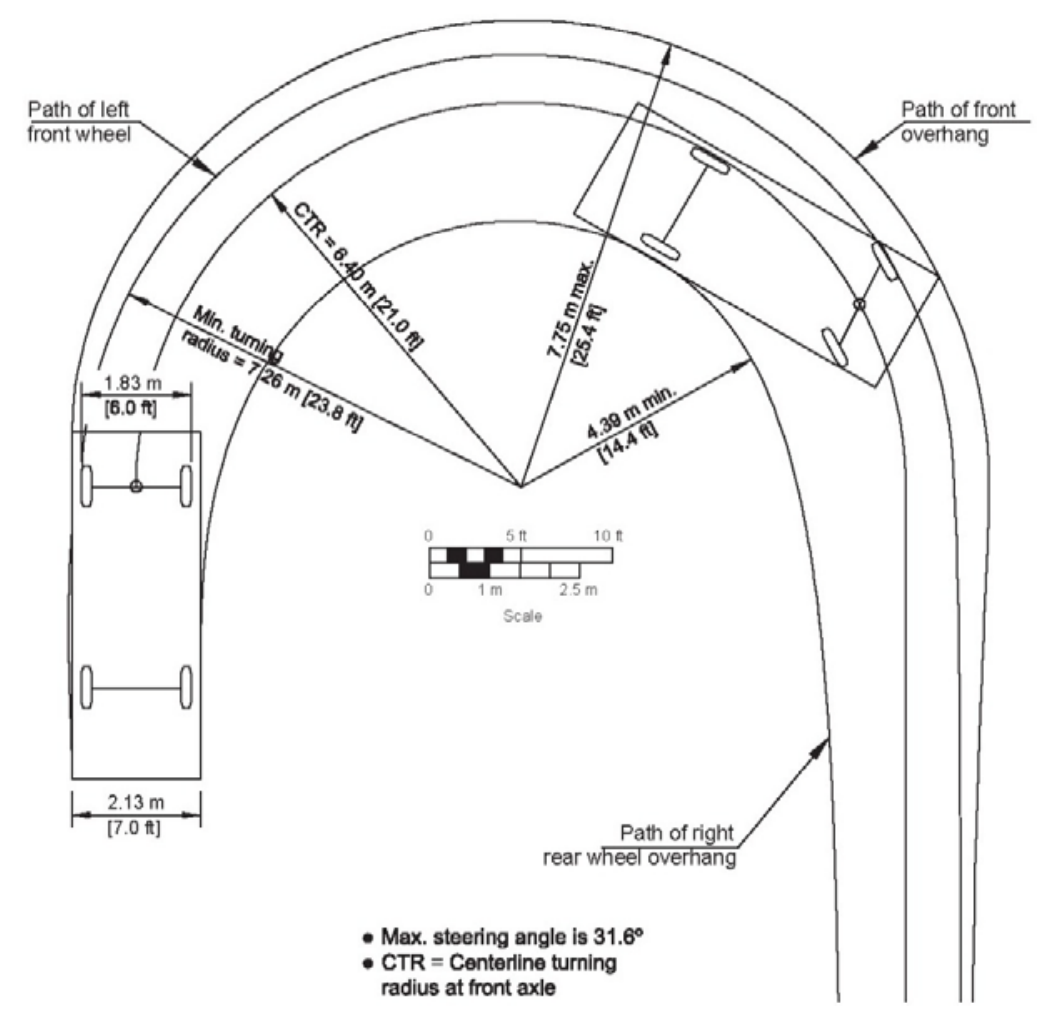


Figure 1. Minimum Turning Path for Passenger Car (P) Design Vehicle Based on the median width, the proposed model consider three cases for U-turn maneuvers namely; U-turn to the inner lane, U-turn to middle lanes, and U-turn to the outer lane of the major road. Each maneuver type has a certain probability depending on median width and traffic mix, where sum of probabilities' of the three types equals the unity $\left(P_{1}+P_{2}+P_{3}=1\right)$.

\subsection{Experimental Design}

The experimental design included input parameters (factors) and the output performance measures (responses). The experimental factors were U-turn maneuver, critical gap, and conflicting traffic. The experimental response was U-turn capacity. A factorial design with 63 design points was considered as shown in Table 1. Three levels of critical gaps, three levels of U-turn maneuvers, and seven levels of conflicting traffic were considered. These levels of v/c are indications for traffic level of services. Levels of the critical gap were 4.0, 5.0, and 6.0 seconds which are equivalent to the conflicting vehicle's speed of 30 , 50, and $70 \mathrm{~km} / \mathrm{hr}$, respectively [6].

Table 1. Matrix for the Factorial Design of the Simulation Experiment

\begin{tabular}{|l|c|l|}
\hline Factor & $\begin{array}{c}\text { No. of } \\
\text { levels }\end{array}$ & Levels \\
\hline Factor 1: U-turn Maneuver & 3 & $\begin{array}{l}\bullet \text { U-turn to the inner lane } \\
\bullet \quad \text { U-turn to the middle lane } \\
\bullet \quad \text { U-turn to the outer lane }\end{array}$ \\
\hline Factor 2: Critical gap (seconds) & 3 & $4.0,5.0$, and 6.0 \\
\hline $\begin{array}{c}\text { Factor 3: Conflicting traffic } \\
\text { (veh / hr / lane) }\end{array}$ & 7 & $\begin{array}{l}100,250,500,750,1000,1250, \\
1500\end{array}$ \\
\hline
\end{tabular}

The Visual Basic.NET (VB.NET) programming language was utilized to build the proposed simulation model. A discussion of data structures and algorithms associated with VB.NET can be found in [9]. 


\section{Model verification}

Model verification is the process of examining the conceptual aspects of the model to ensure it works logically. Verification included tracking vehicles to ensure movements follow the logical sequence built in the model. Model validation is considered to the process of determining to what extent the model's underlying fundamental rules within relevant theories. To verify the proposed simulation model, it was examined against the following mathematical equation that was developed based on the probability of finding a gap of length $T_{c}$ in a single-lane conflict with headways following a negative exponential distribution [10].

$$
C_{p}=v_{c} \frac{e^{-v_{c} T_{c}}}{1-e^{-v_{c} T_{f}}}
$$

where $C_{p}$ is the potential capacity of the U-turn movement (veh/hr), $v_{c}$ is the traffic volume of the major traffic stream (veh/hr), $T_{c}$ is the critical gap, and $T_{f}$ is the followup headway. Note this mathematical equation is limited to a single-lane conflicting flow, deterministic single values for the critical gap and the follow-up time, and the headways in the major stream have a negative exponential distribution. The proposed simulation model can handle any combination of distributions for number of lanes and the headways in the major stream, logic of the U-turn drivers' decision making, and the heterogeneity in critical gaps and follow-up times among the U-turn vehicles. Thirty (30) simulation runs were conducted and the 95\% confidence interval for the true capacity was calculated and revealed no significant difference exists between the simulation model and the above mathematical equation.

\section{Simulation Results and Discussion}

For each of the factor combination, 30 simulation runs were conducted and potential capacity was calculated. For each simulation run, the length of the simulation run was 65 minutes including 5 minutes warm-up period. During the warm- 
up period, results are not collected in order to reduce bias estimate in model results. Runs were conducted for a 3-lane road.

Figure 1 summarizes model results in terms of box plots. A box plot is a graphical representation of the quantile summary. Fifty percent of the results fall within the boundaries of the box. The figure shows significant effect of median width on capacity of U-turns. Table 2 summarizes model results U-turn capacity at different levels of critical gaps, the conflicting traffic volume, and the different U-turn maneuvers. The relationship between U-turn capacity and the conflicting traffic volumes for different median widths is described in Figure 2 through Figure 4. According to Figure 2, the Uturn movement at a wide median can reach a maximum capacity of about $985 \mathrm{veh} / \mathrm{hr}$ when there is no conflicting traffic. The capacity of U-turn decreases as the conflicting volume increases. The capacity is reduced to $540 \mathrm{veh} / \mathrm{hr}$ and $380 \mathrm{veh} / \mathrm{hr}$ when the conflicting volume is 1000 and $1500 \mathrm{veh} / \mathrm{hr}$ per lane.

Table 2. Summary of Model Results for Capacity of U-turn

\begin{tabular}{|c|c|c|c|c|c|c|c|}
\hline & \multicolumn{7}{|c|}{ Conflicting traffic (vehicle / hour / lane) } \\
\hline $\begin{array}{c}\text { Critical gap } \\
\text { (sec) }\end{array}$ & $\mathbf{1 0 0}$ & $\mathbf{2 5 0}$ & $\mathbf{5 0 0}$ & $\mathbf{7 5 0}$ & $\mathbf{1 0 0 0}$ & $\mathbf{1 2 5 0}$ & $\mathbf{1 5 0 0}$ \\
\hline \multicolumn{8}{|c|}{ (a) Wide median width: U-turn to the inner lane of the major road } \\
\hline $\mathbf{4}$ & 985 & 897 & 760 & 638 & 540 & 448 & 380 \\
\hline $\mathbf{5}$ & 955 & 837 & 661 & 519 & 410 & 316 & 257 \\
\hline $\mathbf{6}$ & 934 & 777 & 576 & 423 & 308 & 229 & 171 \\
\hline \multicolumn{7}{|c|}{ (b) Medium median width: U-turn to the middle lane of the major road } \\
\hline $\mathbf{4}$ & 927 & 760 & 537 & 374 & 261 & 174 & 124 \\
\hline $\mathbf{5}$ & 874 & 657 & 407 & 247 & 149 & 91 & 62 \\
\hline $\mathbf{6}$ & 831 & 578 & 312 & 163 & 89 & 54 & 32 \\
\hline (c) Narrow median width: U-turn to the outer lane of the major road \\
\hline $\mathbf{4}$ & 868 & 641 & 373 & 213 & 121 & 65 & 39 \\
\hline $\mathbf{5}$ & 798 & 514 & 248 & 118 & 55 & 37 & 32 \\
\hline $\mathbf{6}$ & 736 & 424 & 165 & 65 & 30 & 28 & 26 \\
\hline
\end{tabular}



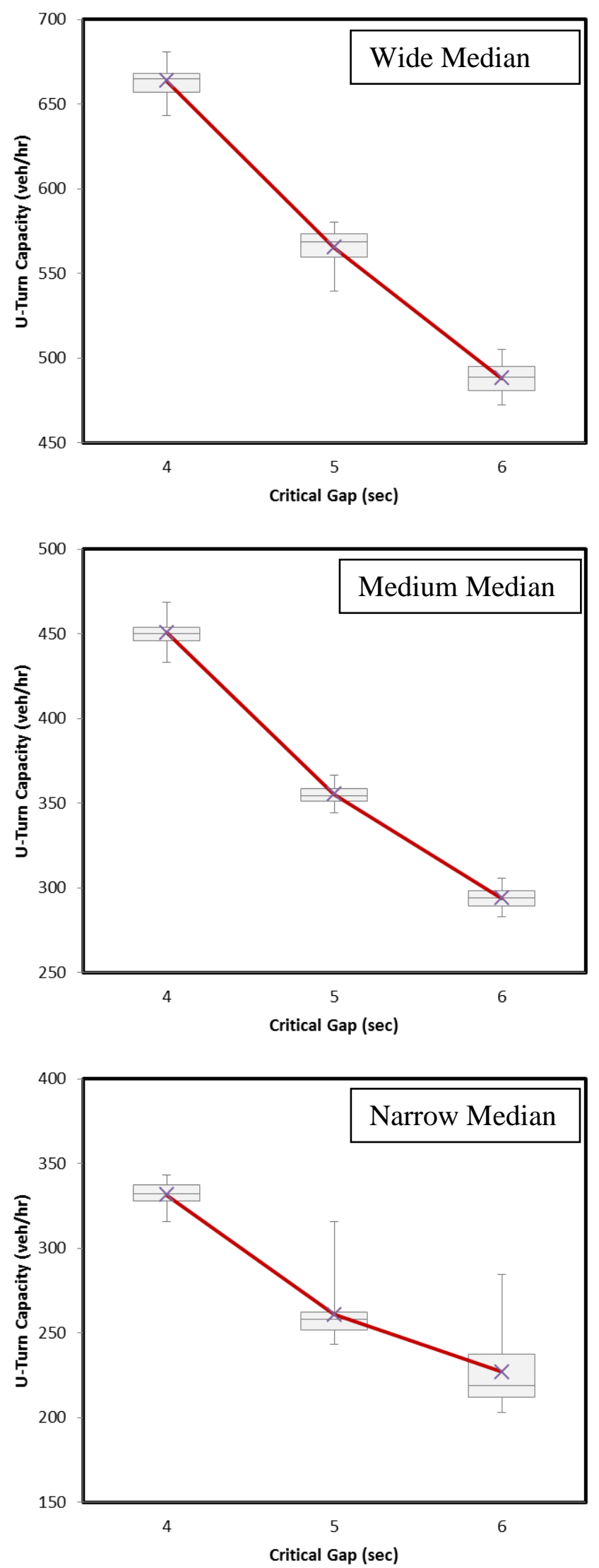

Figure 1. Box Plots for Model Results of The U-turn Capacity 


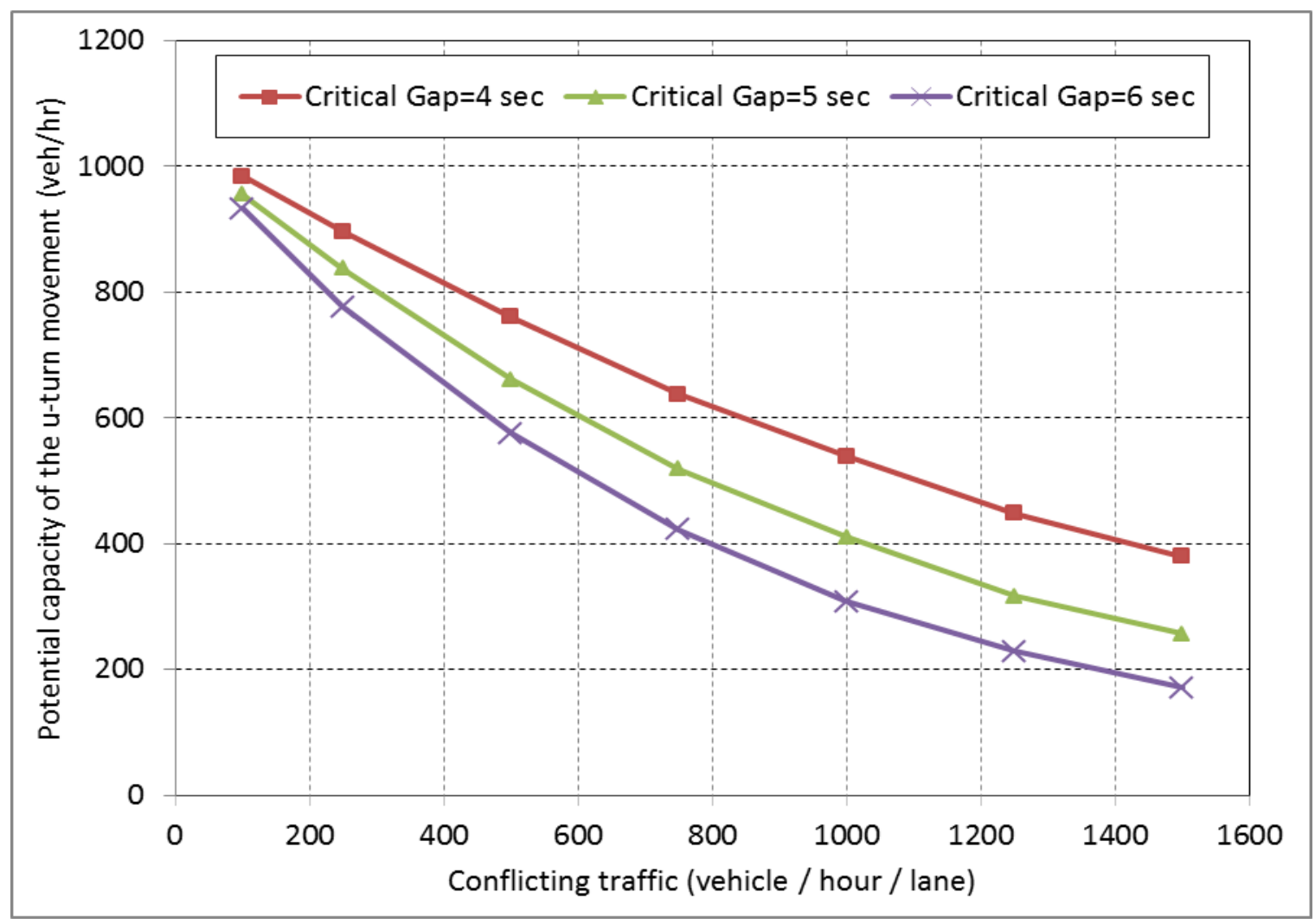

Figure 2. U-Turn Capacity and Conflicting Taffic Relationship, Case of Wide Median

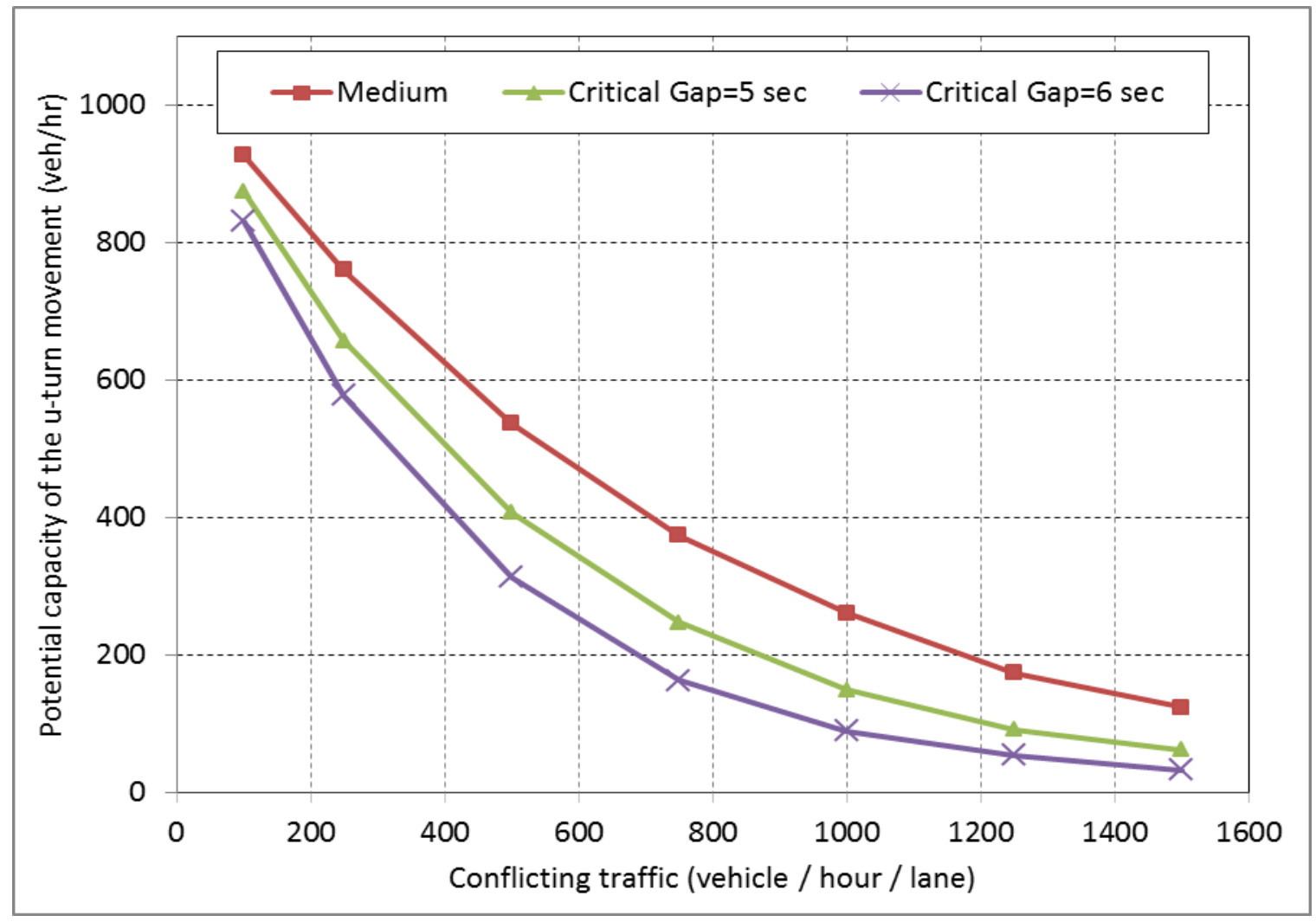

Figure 3. U-Turn Capacity and Conflicting Taffic Relationship, Case of Medium Median 


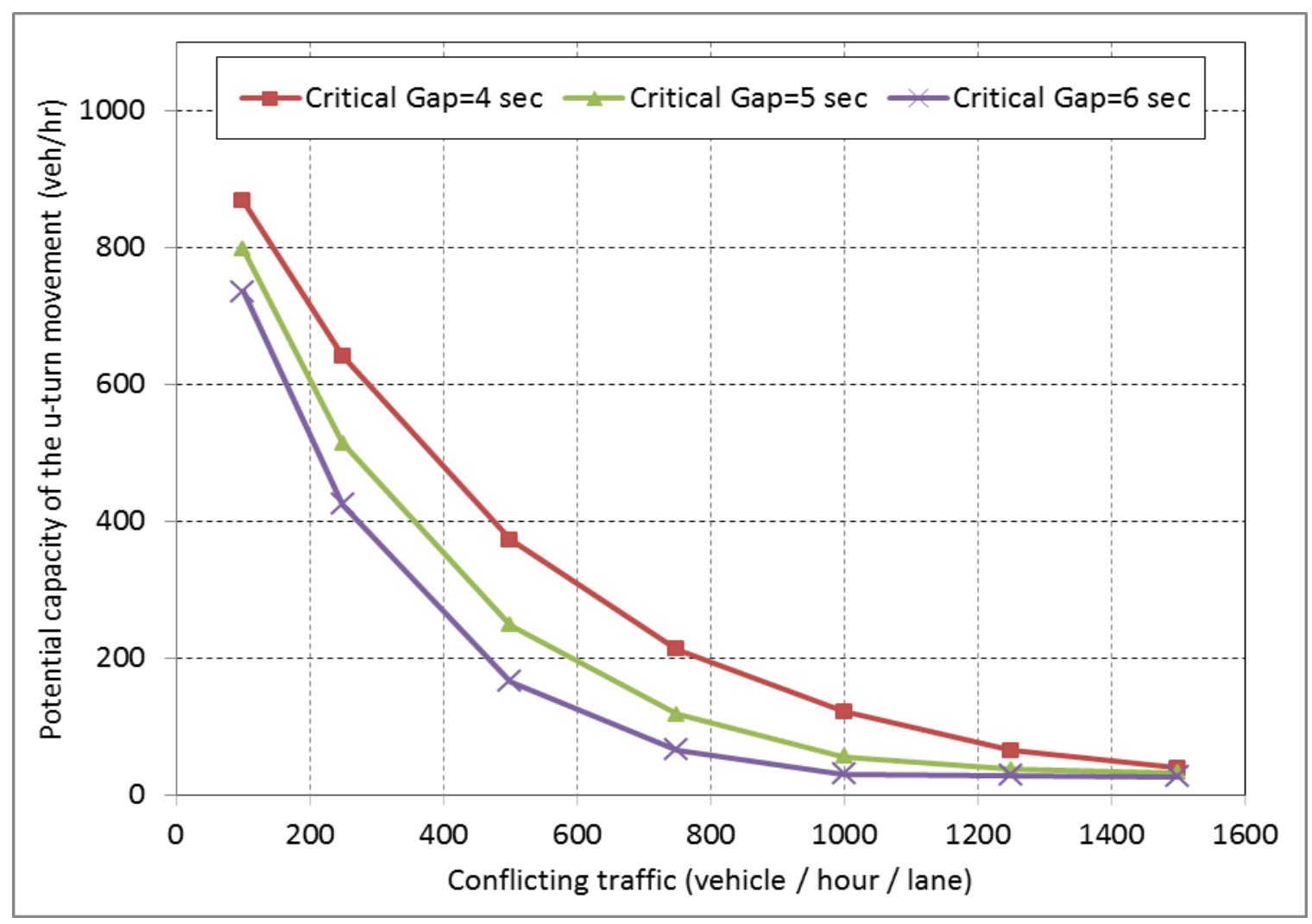

Figure 4. U-Turn Capacity and Conflicting Taffic Relationship, Case of Narrow Median

A metamodel for post-simulation analysis was estimated. The term "metamodel" refers to the use of a mathematical model such as a regression model, constructed from simulation data and used to aid the analysis of the simulated system. The purpose of the metamodel is to approximate the response surface, which in this case is the average speed upstream the microbus stop. Then, the metamodel can be used to learn about how the response (U-turn capacity) would behave over various regions of the inputfactor space (critical gaps, conflicting traffic, and median widths), to estimate how the response would change at a particular point if the input factors were changed slightly, or to find approximately optimal settings of the input factors. The relationship between the response variable $Y$ (U-turn capacity) and the predictor variables $X$ (critical gaps, conflicting traffic, and median widths) can be postulated as the following linear regression model.

$$
Y=\beta_{o}+\beta_{1} X_{1}+\beta_{2} X_{2}+\beta_{3} X_{3}+\beta_{4} X_{4}+\varepsilon
$$


where $\beta_{o}, \ldots, \beta_{4}$ are the model regression coefficients to be estimated as $\hat{\beta}_{o}, \ldots, \hat{\beta}_{4}$, and $\varepsilon$ is a random disturbance or error. In particular $\varepsilon$ contains no systematic information for determining $Y$ that is not already captured in $X$. Table 3 summarizes results of the estimated multiple linear regression metamodel based on the simulation results for speeds upstream the microbus stop. Note the three types of medians were represented by two indicator variables for the narrow and median cases. An indicator variable is an artificial variable created to represent an attribute with two or more distinct categories/levels. The estimated coefficient of the critical gap indicates that an increase of 1-second in critical gap would reduce the U-turn capacity by an average of about $75 \mathrm{veh} / \mathrm{hr}$. The quality of the fit can be assessed by the larger $t$-statistic (in absolute value) or the smaller corresponding $p$-value, the stronger the linear relationship between $Y$ and $X$. Also in linear regression, $R^{2}$ is used to describe the model goodness of fit between the average speed upstream the microbus stops ( $Y$ response variable) and (v/c) ratio and microbus headway time (the predictor variables $X$ ). The value $R^{2}=0.90$ indicates that nearly $90 \%$ of the total variability in the response variable (U-turn capacity) is accounted for by the predictor variables (critical gaps, conflicting traffic, and median widths). The high value of $R^{2}$ indicates a strong linear relationship between the U-turn capacity and critical gaps, conflicting traffic, and median widths

Table 3. U-turn Capacity Estimation Model

\begin{tabular}{|c|c|c|c|c|c|}
\hline \multicolumn{2}{|l|}{ Predictor } & Coefficient & Standard error & t-stat & p-value \\
\hline \multicolumn{2}{|l|}{$\beta_{o}$} & 1,346 & 83.39 & 16.14 & 0.00 \\
\hline \multicolumn{2}{|l|}{ Critical Gap (sec) } & -75.54 & 15.57 & -4.85 & 0.00 \\
\hline \multicolumn{2}{|c|}{ Conflicting Traffic (veh/hr/lane) } & -0.52 & 0.03 & -19.54 & 0.00 \\
\hline \multicolumn{2}{|c|}{$\begin{array}{l}\text { Narrow Median Indicator (1 for } \\
\text { narrow median, } 0 \text { otherwise) }\end{array}$} & -305.87 & 31.14 & -9.82 & 0.00 \\
\hline \multicolumn{2}{|c|}{$\begin{array}{l}\text { Medium Median Indicator ( } 1 \text { for } \\
\text { medium median, } 0 \text { otherwise) }\end{array}$} & -205.73 & 31.14 & -6.61 & 0.00 \\
\hline \multicolumn{6}{|c|}{ Standard Error $=100.89 \quad R^{2}=0.90$} \\
\hline ANOVA & $\mathrm{DF}$ & SS & MSS & F-stat & p-value \\
\hline Model & 4 & 5146741.49 & 1286685.37 & 126.40 & 0.00 \\
\hline Residual & 58 & 590413.75 & 10179.55 & & \\
\hline Total & 62 & 5737155.24 & & & \\
\hline
\end{tabular}




\section{Conclusions}

The paper focused on development of a traffic simulation model to study capacity of U-turns. The developed model was used to analyze different combinations of three factors; critical gaps, median widths, and the conflicting traffic volumes. For each factor combination, 30 simulation runs were conducted and the U-turn capacity was calculated. Then a metamodel for post-simulation analysis was developed to estimate the U-turn capacity as a function of the three considered factors.

Results showed when the median width was comfortably sufficient; it leaded to a higher U-turn capacity. The U-turn movement at a wide median can reach a maximum capacity of about $985 \mathrm{veh} / \mathrm{hr}$ when there is no conflicting traffic. The capacity of Uturn decreases as the conflicting volume increases. The capacity is reduced to 540 veh/hr and $380 \mathrm{veh} / \mathrm{hr}$ when the conflicting volume is 1000 and 1500 veh/hr per lane

\section{References}

1. Nakat, Z., and Herrera, S., Cairo Traffic Congestion Study Phase 1, World Bank, Final Report, November 2010.

2. El-Esawey, M., and Sayed, T., Operational performance analysis of the unconventional median U-turn intersection design, Canadian Journal of Civil Engineering, Vol. 38, 2011.

3. Al-Masaeid, H., Capacity of U-turns at Median Openings, ITE Journal, Vol. 69, No. 6, 1999.

4. Aldian, A., and Taylor, M., Selecting Priority Junction Traffic Models to Determine Uturn Capacity at Median Opening, Proceedings of the Eastern Asia Society for Transportation Studies, Vol. 3, No. 2, 2001.

5. Jenjiwattankul, T., Sano, K., and Nishiuchi, H., Capacity of U-turn at Midblock Median Opening on Urban Arterial Based on Balancing volume-to-capacity ratio, Proceedings of the Eastern Asia Society for Transportation Studies, Vol. 9, 2013.

6. Jenjiwattankul, T., Traffic capacity analysis on U-turn at midblock median openings on urban arterials, PhD Dissertation, Nagaoka University of Technology, August 2013.

7. Garber, N., and Hoel, L., Traffic and Highway Engineering, Fourth Edition, Cengage Learning, 2009.

8. Chung, C., Simulation Modeling Handbook: A Practical Approach, Industrial and Manufacturing Engineering Series, CRC Press, 2004.

9. McMillan, M., Data Structure and Algorithms Using Visual Basic.NET, Cambridge University Press, 2005.

10. Baass, K., The Potential Capacity of Unsignalized Intersections, ITE Journal, October 1987.

11. May, A., Traffic Flow Fundamentals, Prentice-Hall, Inc., 1990. 